\title{
Lafora disease fibroblasts exemplify the molecular interdependence between thioredoxin 1 and the proteasome in mammalian cells.
}

José Luis García-Giménez ${ }^{\text {a,b,c }}$, Marta Seco-Cervera a, Carmen Aguado ${ }^{\text {a,e }}$, Francisco Dasí $^{\text {b,c }}$, Carlos Romá-Mateo a,f, Sonia Priego d , Jelena Markovic ${ }^{\mathrm{d}}$, Erwin Knecht, ${ }^{\mathrm{a}, \mathrm{e}}$ Pascual Sanz ${ }^{\text {a,f }}$, Federico V. Pallardó ${ }^{\text {a,b,c }}$

${ }^{a}$ CIBERER. Centro de Investigación Biomédica en Red de Enfermedades Raras. Valencia. Spain.

${ }^{\mathrm{b}}$ FIHCUV-INCLIVA. Valencia. Spain

${ }^{\mathrm{c}}$ Dept. Physiology. Medicine School. University of Valencia. Valencia. Spain

${ }^{\mathrm{d}}$ Research core facilities of Medicine School. University of Valencia. Valencia. Spain

${ }^{\mathrm{e}}$ CIPF. Centro de Investigación Príncipe Felipe. Valencia. Spain.

${ }^{\mathrm{f}}$ IBV-CSIC. Instituto de Biomedicina de Valencia. Consejo Superior de Investigaciones Científicas. Valencia. Spain.

Corresponding Author:

PhD. MD. Federico V. Pallardó

Dpt. Physiology. Medicine School.

University of Valencia.

Av/ Blasco Ibañez, 15, 0.

46010, Valencia (Spain)

Phone: 0034963864641

FAX: 0034963864642 


\begin{abstract}
Thioredoxin 1, Trx1 is a key regulator of cellular redox balance and participates in cellular signaling events. Recent evidence in yeast have shown that members of the Trx family interact with the $20 \mathrm{~S}$ proteasome, indicating redox regulation of proteasome activity. However, there is little information about the interrelationship of Trx proteins with the proteasome system in mammalian cells, especially in the nucleus.
\end{abstract}

Here, we have studied this relationship in different cellular conditions of mammalian cells. We have showed that Trx1 levels and its subcellular localization (cytosol, endoplasmic reticulum and nucleus) depend on the proteasome activity during cell cycle in NIH3T3 fibroblasts or stress conditions, when proteasome was inhibited. In addition, we also studied how the main cellular antioxidant systems are stimulated when proteasome activity is inhibited in NIH3T3 cells. Finally, we describe how Trx1 levels are decreased in Lafora fibroblasts. In addition, nuclear co-localization of Trx 1 with $20 \mathrm{~S}$ proteasome in laforin deficient cells was altered compared with control cells. Our results indicate a close relationship between $\operatorname{Trx} 1$ and 20S nuclear proteasome and give a new perspective to study diseases or physiopathological conditions in which defects in the proteasome system are associated to oxidative stress.

\title{
Keywords
}

Thioredoxin 1, proteasome, cell proliferation, Lafora disease, antioxidant enzymes, rare diseases 


\section{Introduction}

Classically, the thioredoxin system has been considered a general disulfide reductase catalyzing NADPH-dependent reduction of S-S bridges in oxidized proteins. Thioredoxin (Trx) was also known as a hydrogen donor for ribonucleotide reductase, the essential enzyme that provides deoxyribonucleotides for DNA replication $[1,2]$. However, nowadays Trx is known to be a multifunctional protein that, depending on the isoform or cellular stimuli $[3,4]$, has distinct roles in each of cellular locations $[5,6]$. Thus, in the cytoplasm, Trx can regulate the redox environment of the cell and also the activity of certain proteins, such as the apoptosis signal-regulating kinase-1 (ASK-1) and hence controlling apoptosis $[7,8]$. In the case of Trx 1 , it has been reported that it is localized mainly in the cytoplasm, although several factors such as radical oxygen species (ROS), ionizing radiations or UV light are able to induce the migration of Trx1 to the nucleus, despite the lack of a clear nuclear localization signal [3]. The nuclear translocation of Trx1 may be associated with the signaling molecules that connect the cytoplasm and the nucleus events [3], and suggests that Trx1 may be involved in nuclear processes. In fact, it has been described that Trx1 interacts with many transcription factors and thereby regulates gene expression $(5,8,9)$.

Proteasomes are responsible for most of the selective degradation of nuclear and cytosolic proteins $[9,10]$ and, therefore, control many essential processes such as cell proliferation, cell-cycle progression, transcriptional regulation, regulation of cell signalling, the unfolded protein response (UPR), and endocytosis $[4,11]$.

Furthermore, a decrease in proteasome activity has been related with common diseases such as Alzheimer [12], Parkinson [13] and with rare diseases such as amyotrophic lateral sclerosis [14], Huntington disease [15] and Lafora disease, a fatal form of 
progressive myoclonus epilepsy [16]. Interestingly, proteasome activity is also decreased during aging, as reviewed by Low et al. [17].

Recently, a direct link between proteasome activity and Thioredoxin-interacting protein (TrxINP) has been reported [18], and other authors have established an interaction of Txnl1 with some proteasome subunits in yeast $[19,20]$.

Thus, to gain a deeper insight into the relationship between Trx1 and proteasome activity in mammalian cells, we have used NIH3T3 fibroblasts to analyze changes in Trx1 and proteasome activity during cell cycle and the effect of the down-regulation of the proteasome activity in the expression of Trx1 and their subcellular localization. In this work we provide evidence for a relationship between proteasome activity and Trx1 regulation and define pathways that could play important roles in the regulation of antioxidant enzymes and in the control of apoptosis.

In addition, we have also analyzed the pathophysiological consequences of this interrelationship in a disease in which proteasomal activity is decreased; Lafora disease. In fibroblasts from patients suffering this disease we have observed changes in Trx1 protein levels and also in the subcellular location of $\operatorname{Trx} 1$ and proteasomes, thus confirming a molecular interaction between both. 


\section{Material and Methods}

\section{Cell culture}

NIH3T3 cells were grown in Iscove's modified Dulbecco's medium supplemented with $10 \%$ foetal bovine serum and $1 \%$ penicillin/streptomycin at $37{ }^{\circ} \mathrm{C}$ and $5 \% \mathrm{CO} 2$. Growth curves in NIH3T3 fibroblasts were obtained using a hemocytometer chamber at 6, 24, 48 and $120 \mathrm{~h}$ after plating.

Laforin-deficient fibroblasts were obtained from two patients with the clinical features of Lafora disease and with two different mutations, Y86X (Laf-1) and R241X (Laf-2), in the laforin gene (EPM2A) and control fibroblasts were obtained from healthy subjects matched by sex and age. Controls were kindly donated by Dra. Del Rio from the CIEMAT (Madrid, Spain) and Dra. Amparo Gimeno Universitat de València (Valencia, Spain). Fibroblasts were cultured at $37{ }^{\circ} \mathrm{C}$ in a humidified $5 \% \mathrm{CO}_{2}$ atmosphere in Dulbecco's modified Eagle's medium supplemented with $15 \%$ foetal bovine serum, $1 \%$ glutamine, 100 units $/ \mathrm{mL}$ penicillin and $100 \mu \mathrm{g} / \mathrm{mL}$ streptomycin (all from Invitrogen Life Technologies).

\section{Measurements of DNA synthesis}

Proliferation of cell lines was determined with the "Cell proliferation ELISA BrdU colorimetric assay" from Roche. Cells were cultured in 96 wells plates at 6, 24, 48 and $120 \mathrm{~h}$. The final reaction was measured with the spectrophotometer Spectra MAXPLUS 384 from Molecular Devices, at wavelengths of 370-492 nm and 3 intervals of $5 \mathrm{~min}$. The variation of absorbance is proportional to the incorporation of BrdU. This value was divided at each time by the total cell content, determined with the sulforhodamine B assay based on the spectrophotometric measurement of cellular protein [21]. 
RNA isolation and cDNA synthesis

Total RNA was isolated from cells using the PARIS ${ }^{\mathrm{TM}}$ Protein and RNA Isolation System (Ambion, Austin, TX, USA) according to the manufacturer's instructions. For reverse transcription reactions (RT), $1 \mathrm{mg}$ of the purified RNA was reverse transcribed using random hexamers with the High-Capacity cDNA reverse transcription kit (Applied Biosystems, P/N: 4322171; Foster City, CA, USA) according to the manufacturer's instructions. RT conditions comprised an initial incubation step at $25^{\circ} \mathrm{C}$ for $10 \mathrm{~min}$ to allow random hexamers annealing, followed by cDNA synthesis at $37^{\circ} \mathrm{C}$ for $120 \mathrm{~min}$, and a final $5 \mathrm{~min}$ inactivation step at $95^{\circ} \mathrm{C}$.

\section{Measurement of mRNA Levels}

The mRNA levels were determined by quantitative real-time PCR analysis using an ABI Prism 7900 HT Fast Real-Time PCR System (Applied Biosystems, Foster City, CA). Gene-specific primer pairs and probes for TRX1 (Thioredoxin 1) (Assay-ondemand, Applied Biosystems), were used together with TaqManH Universal PCR Master Mix (Applied Biosystems, P/N 4304437, Foster City, CA) and reverse transcribed sample RNA in $20 \mu \mathrm{l}$ reaction volumes. PCR conditions were $10 \mathrm{~min}$ at $95^{\circ} \mathrm{C}$ for enzyme activation, followed by 40 two-step cycles $\left(15 \mathrm{sec}\right.$ at $95^{\circ} \mathrm{C} ; 1 \mathrm{~min}$ at $\left.60^{\circ} \mathrm{C}\right)$. The levels of glyceraldehyde-3-phosphate dehydrogenase expression were measured in all samples to normalize results differences in RNA input, RNA quality and reverse transcription efficiency. Each sample was analyzed in triplicate, and the expression was calculated according to the $2^{-\Delta \Delta \mathrm{Ct}}$ method [22]. 


\section{Total protein and histone extraction}

Total protein extracts were obtained from approximately $10^{6}$ cells lysed in ice for 15 min using $100 \mu \mathrm{L}$ of lysis buffer (20 mM Hepes, $\mathrm{pH}$ 7.4, 1\% Triton X-100, $100 \mathrm{mM}$ $\mathrm{NaCl}, 50 \mathrm{mM}, \mathrm{NaF}, 10 \mathrm{mM}, \quad \beta$-glycerophosphate, $1 \mathrm{mM}$ activated sodium orthovanadate, $1 \mathrm{mM}$ PMSF and $2 \mu \mathrm{L} / \mathrm{mL}$ protein proteases inhibitor cocktail). Then, the suspension was spun-down at $13000 \mathrm{~g}$ for $10 \mathrm{~min}$ at $4{ }^{\circ} \mathrm{C}$ and supernatants were collected and stored at $-80^{\circ} \mathrm{C}$ until use.

Nuclei and histones for gamma-H2AX analysis were isolated as described previously [23]. Protein content was determined by a modified Lowry's method [24].

\section{Western blot analysis}

Aliquots of cell lysates (30 $\mu$ g protein) were added to sample buffer containing $10 \% \beta$ mercaptoethanol and the mixture was immediately boiled for 5 min. Proteins were separated by electrophoresis in sodium dodecyl sulfate-polyacrylamide (12\%) gels (SDS-PAGE), at $100 \mathrm{~V}$ during $2 \mathrm{~h}$. After electrophoresis, the proteins were electroblotted (Bio-Rad) onto nitrocellulose membranes. Membranes were blocked with $0.05 \mathrm{~g} / \mathrm{ml}$ non-fat milk or with $0.05 \mathrm{~g} / \mathrm{ml}$ BSA in TBS containing $0.2 \%$ Tween 20 (TBST), depending on the antibody, washed three times at room temperature, and incubated for $2 \mathrm{~h}$ at room temperature with primary antibodies diluted in TBST with $0.01 \mathrm{~g} / \mathrm{ml}$ non-fat milk as follows: antioxidant enzymes as previously used [23], Trx1 (1:1000, Abcam), TrxR1 (1:1000, Abcam), Trx2, Grx1 (1:1000, Abcam), Grx2 (1:1000, Bax, Bid and Bim (1:1000, GeneTex), ubiquitin-protein conjugates (1:1000, Enzo Life Sciences), PDI (1:1000, Cell Signaling), BIP (1.1000, Cell Signaling), ATF4 (1.1000, Santa Cruz Techonologies), CHOP (1.1000, ), c-myc (, ), Rpn7 $\alpha$ (1:1000, ENZO). As loading controls, antibodies that recognize $\alpha$-tubulin or $\beta$-actin $(1: 1000$, Santa Cruz 
BioTech. USA) antibodies were used. Thereafter, the blots were washed again with TBST and further incubated for $1 \mathrm{~h}$ with a secondary mouse, rabbit or goat antibody conjugated with horseradish peroxidase. Blots were incubated with $\mathrm{ECL}^{\mathrm{TM}}$ Western Blotting Detection Reagents as specified by the manufacturer (Amersham GE HealthcareBio-Sciences AB, Uppsala, Sweden). Chemioluminescent signals were assessed using a General Electric's scanning densitometer (LAS-4000).

Preparation of recombinant c-myc-Trx1 and Co-Immunoprecipitation

Preparation of recombinant proteins for Co-IP assays was performed using E. coli. Briefly,

The protein extracts containing c-myc-Trx1 (corresponding to three $100 \mathrm{~cm}^{2}$ dishes of cultured NIH3T3) were allowed to bind to sepharose beads overnight at $4{ }^{\circ} \mathrm{C}$. Beads were then washed twice with PBS and twice with binding buffer $(50 \mathrm{mM}$ Tris- $\mathrm{HCl}, \mathrm{pH}$ 8, $150 \mathrm{mM} \mathrm{NaCl}, 5 \mathrm{mM}$ EDTA, 0.5\% Nonidet P-40, and protease inhibitor mixture). The beads were then incubated for $45 \mathrm{~min}$ at room temperature under gentle rotation in $200 \mu \mathrm{l}$ of binding buffer containing $25 \mu \mathrm{g}$ of BSA and $20 \mu \mathrm{g}$ of purified protein extracs. Beads were recovered by centrifugation, washed six times in the binding buffer, resuspended in SDS-PAGE buffer, and loaded on a 12\% denaturing polyacrylamide gel.

\section{Confocal microscopy}

Confocal images were acquired using a Leica TCS-SP2 confocal laser scanning unit equipped with argon and helium-neon laser beams and attached to a Leica DM1RB inverted microscope (Leica Microsystems, Mannheim, Germany). NIH3T3 fibroblasts were plated in $2 \mathrm{~cm}^{2}$ LAB-TEK II chambered cover glass (Nunc, Thermo Fischer Scientific, Waltham, MA, USA) and maintained in culture for 24 and $48 \mathrm{~h}$ before adding or not $0.2 \mu \mathrm{M} \mathrm{MG132.}$ 
All treatment conditions and controls were processed and analyzed on the same day. Cells were fixed in $4 \%$ formaldehyde, washed in phosphate buffered saline (PBS) and permeabilized with PBS containing $0.5 \%$ Triton X-100 for 20 min, washed twice with PBS and blocked in $2 \%$ BSA during $1 \mathrm{~h}$ at room temperature. Then, immunostaining was carried out, incubating the cells overnight at $4^{\circ} \mathrm{C}$ first with anti-Trx1 (1:200, Abcam, Cambridge, MA, USA), anti-20S $\alpha 7$ subunit (1:1000, Biomol, Pennsylvania, USA). The next day, the cells were washed twice with PBS and incubated in the dark with goat anti-rabbit secondary antibody Alexa Fluor 543 (1:350, Invitrogen) or Alexa Fluor 488 (1:350, Invitrogen) for $1 \mathrm{~h}$ at room temperature. Finally, nuclei were stained using $2 \mu \mathrm{g} / \mathrm{ml}$ Hoechst (Sigma-Aldrich, St. Louis, MO, USA).

Flow cytometry analysis

Cells $\left(10^{6}\right.$ cells $\left./ 100 \mu \mathrm{L}\right)$ were stained with Annexin V Alexa Fluor ${ }^{\circledR} 488$ Conjugate (Invitrogen Probes) in Annexin V binding buffer (10 mM Hepes, $140 \mathrm{mM} \mathrm{NaCl}, 25$ $\mathrm{mM} \mathrm{CaCl} 2$ ) at room temperature for $15 \mathrm{~min}$. In the last $5 \mathrm{~min}$ the cells were stained also with $60 \mu \mathrm{g} / \mathrm{ml}$ of propidium iodide (Sigma-Aldrich, St. Louis, MO, USA). Single stained controls were prepared for spectral compensation.

Images were obtained on the ImagesStream IS100 Flow cytometer (Amnis Corporation, Seattle, WA) using blue laser $488 \mathrm{~nm}$ excitation at $20 \mathrm{~mW}$. Cell classifier "area lower limit" was set to 100 pixels in the bright field imagery, to avoid the collection of debris with data and to ensure that events of interest were not lost. Typical files contained imagery for 5000-8000 cells using 4 channels: side scatter on channel 1, bright field on channel 2, channel 3 for Annexin V-Alexa fluor 488 staining, and channel 5 for 
propidium iodide staining. Quantitative measurements were performed using IDEAS ${ }^{\circledR}$ 4.0 (Image Data Exploration and Analysis Software). Compensated image files were created using the compensation matrix based on single stained controls acquired with no-bright field option. Subsequently, doublets and debris and cells out of focus were excluded from analysis using typical IDEAS features (Aspect ratio intensity vs. Area for channel 2, and gradient RMS for channel 2, respectively). On the dot blot created for Annexin V-Alexa fluor 488 vs PI staining, four cell populations were discriminated and gated: live cells: low presence and intensity of Annexin V-Alexa fluor 488 and PI fluorescence negative, dead cells: high PI staining and low Annexin V-Alexa fluor 488 staining, cells in early apoptosis: high intensity and presence of Annexin V-Alexa fluor 488 staining, and finally cells in late apoptosis: double positive cells, stained both with high Annexin V-Alexa fluor 488 and PI fluorescence. The same gating criteria were applied to all the samples, and the results from 2 experiments are presented in \% vs. control, with the corresponding SD.

\section{Proteasome activities}

Proteasome activities were measured spectrofluorometrically using synthetic fluorogenic peptides. Human fibroblasts from controls and Lafora disease patients were incubated under high (Krebs-Henseleit medium: $118.4 \mathrm{mM} \mathrm{NaCl}, 4.75 \mathrm{mM} \mathrm{KCl}, 1.19$ $\mathrm{mM} \mathrm{KH}{ }_{2} \mathrm{PO}_{4}, 2.54 \mathrm{mM} \mathrm{MgSO} 4,2.44 \mathrm{mM} \mathrm{CaCl}_{2} .2 \mathrm{H} 2 \mathrm{O}, 28.6 \mathrm{mM} \mathrm{NaHCO}, 10 \mathrm{mM}$ glucose with $10 \mathrm{mM}$ Hepes, $\mathrm{pH}$ 7.4) and low (full medium) proteolysis conditions for 3

h. Then, cells were washed two times with PBS and scraped from the culture plates in an ice-chilled buffer containing $50 \mathrm{mM}$ Hepes pH 7.4, $150 \mathrm{mM} \mathrm{NaCl,} 5 \mathrm{mM}$ EDTA, 10 $\mu \mathrm{M}$ leupeptin, $1 \mu \mathrm{g} / \mathrm{ml}$ pepstatin and $1 \mathrm{mM}$ phenylmethylsulfonyl fluoride. Homogenization was performed by five cycles of freezing $\left(-80{ }^{\circ} \mathrm{C}\right)$ and thawing at 37 
${ }^{\circ} \mathrm{C}$. Crude extracts were centrifuged at 3,000 g for 10 min at $4{ }^{\circ} \mathrm{C}$. Supernatants were used to measure proteasome peptidase activities in a microplate reader with the following synthetic fluorogenic peptides: N-Suc-LLVY-MCA $\left(\begin{array}{lll}50 & \mu \mathrm{M}\end{array}\right)$ for chymotrypsin-like activity, Boc-LRR-MCA $(50 \mu \mathrm{M})$ for trypsin-like activity and ZLLE-MCA $(50 \mu \mathrm{M})$ for caspase-like activity. In all cases, activity was measured under linear conditions. As controls, parallel reactions were run containing epoxomicin (10 $\mu \mathrm{M})$ or lactacystin $(10 \mu \mathrm{M})$. Protein was measured by a Lowry-deoxycholate procedure.

\section{Pulse-chase experiments}

Fibroblasts from controls and Lafora disease patients were cultured as described under Cell cuture. To label long-lived proteins, fibroblast were incubated for $48 \mathrm{~h}$ in fresh full medium containing $2 \mu \mathrm{Ci} / \mathrm{ml}\left[{ }^{3} \mathrm{H}\right]$ valine (Hartmann Analytic Gmbh), followed by a $24 \mathrm{~h}$ chase in fresh full medium plus $10 \mathrm{mM}$ L-valine to degrade short-lived proteins [25]. Then, all cultures were washed and incubated for $4 \mathrm{~h}$ in Krebs-Henseleit medium (under high proteolysis conditions) with $10 \mathrm{mM}$ Hepes, $\mathrm{pH}$ 7.4, containing $10 \mathrm{mM}$ valine and the indicated additions. Intracellular protein degradation, which was analysed $1 \mathrm{~h}$ later to ensure maximal effects of the additions and for only $3 \mathrm{~h}$ to avoid possible secondary effects, was calculated at intervals of $1 \mathrm{~h}$ by measuring the net release of trichloroacetic acid-soluble radioactivity from the labelled cells into the culture medium and expressed as percentage of protein degraded per $\mathrm{h}$. The contribution of proteasomes to intracellular protein degradation was calculated using $10 \mu \mathrm{M}$ lactacystin as previously described [26]

\section{Statistical analysis}

For the statistical analysis of the results, the mean was taken as the measurement of the main tendency, while standard deviation (SD) was taken as the dispersion measurement. 
A one way analysis of variance was used to determine the difference between groups for DNA synthesis and expression analysis of Trx 1 gene. When an interaction effect was found, multiple comparisons using the Student-Newman-Keuls method post hoc test were performed. When only two groups were analyzed, the one-tailed Student's t-test was used. Each measurement was performed using independent experiments (for specific details, see the corresponbding text or legend to the figure). The alpha level for statistical significance was set at $p<0.05$.

\section{Results}

During DNA synthesis in NIH3T3 fibroblasts the expression of the Trx1 protein increases and co-localizes with the 20 s proteasome

Based on previous results [23], we decided to study the expression and cellular localization profiles of $\operatorname{Trx} 1$ and the $20 \mathrm{~S}$ proteasome in NIH3T3 cells, an established mammalian cell line whose cell cycle, nuclear redox profile and proteasome activity have been extensively characterized by our group [23, 27]. Analysis of the cell proliferation profile in these cells by BrdU showed maximal DNA synthesis at $24 \mathrm{~h}$ of culture and the lowest at $120 \mathrm{~h}$, when cells reached confluence (figure 1A). The expression of the TRX1 gene did not change significantly during these times (figure 1B). However, the levels of the Trx 1 protein increased at 24 and $48 \mathrm{~h}$ of cell culture, compared to initial (6 h) after seeding, but decreased when the cells reached confluence (120 h) (figure 1C). 
Since Trx 1 has been found to localize differently depending on the redox environment or the cell culture conditions [28], we next analyzed the distribution of Trx1 in the cells at different times of cell proliferation and compared it to that of $20 \mathrm{~S}$ proteasomes to identify a possible relationship of these proteins during cell proliferation. Our results (figure 2), indicate that Trx1 cellular distribution of Trx1 changes during cell proliferation. At $6 \mathrm{~h}$ of culture, Trx 1 was mainly perinuclear. At $24 \mathrm{~h}$ and $48 \mathrm{~h}$, a fraction of Trx1 was internalized into the nucleus, showing then a homogenous nuclear/cytosolic distribution. However, at $120 \mathrm{~h}$ of cell culture, just when cells reached confluence, Trx1 was mainly cytosolic. This pattern was very similar to that obtained for the 20S proteasome, which showed a perinuclear distribution at $6 \mathrm{~h}$ (figure 2), a nuclear/cytosolic localization at 24 and $48 \mathrm{~h}$ after cell culture and a redistribution to the cytosol when cells reach confluence at $120 \mathrm{~h}$ (figure 2). Therefore, our results indicate that in NIH3T3 fibroblasts Trx1 and 20S proteasomes follow a similar pattern of subcellular distribution and that both co-localize in the nucleus during cell proliferation.

To confirm the physical interaction of $\operatorname{Trx} 1$ and $20 \mathrm{~S}$ proteasome, expression of c-mycTrx1 protein was induced in NIH3T3 cells. The Co-IP assays confirmed that Trx1 interacts at least with $20 \mathrm{~S}$ through the Rpn7 $\alpha$ subunit (Figure XX).

Changes in protein levels and sub-cellular localization of Trx1 under proteasome inhibition

The results described above indicate that in mammalian cells Trx1 and 20S proteasomes are molecularly connected, as it has been also recently described in yeast [29]. In order to confirm this, we evaluated the effects of proteasome inhibition (using MG132, a N(benzyloxycarbonyl)leucinylleucinylleucinal peptide) on TRX1 gene and protein expression in NIH3T3 fibroblasts. In these experiments, we used a MG132 dose of 0.2 
$\mu \mathrm{M}$, which is lower than the $\mathrm{IC}_{50}$ value (Figure $\mathrm{S} 1 \mathrm{~A}$ ) and did not increase the number of dead cells (Figure S1B). We evaluated the number of cells in early apoptosis, late apoptosis and necrosis, after growing the cells with and without MG132 for 24 and 48 h. Under these conditions, we did not observe any increase in the levels of apoptotic or necrotic cells (figure S2A).

As expected, our results showed that incubation of NIH3T3 with MG132 at $0.2 \mu \mathrm{M}$ for 24 and $48 \mathrm{~h}$ inhibits cellular proteasome activity (figure 3A, upper panel) and the proteasome activity of isolated nuclei from NIH3T3 fibroblasts (figure 3A, lower panel) and produces the accumulation of poly-ubiquitinated proteins (figure 3B). Under these conditions, the DNA synthesis was lower in the MG132-treated cultures (figure 3C), while cell viability, measured with the trypan blue exclusion test, was not affected (data not shown). These results indicate that although the $0.2 \mu \mathrm{M}$ MG132 treatment is nonlethal, it inhibits the synthesis of DNA. Proteasome inhibition also reduced the protein levels of poly(ADP-ribose) polymerase (PARP), an essential enzyme for DNA synthesis progression involved in rejoining the Okazaki's fragments of the lagging strand (figure 3D). Furthermore, our results showed that DNA double strand breaks (DSBs) were increased at 24 and $48 \mathrm{~h}$ after treatment with MG132, as indicated by the increase in gamma-H2AX (figure S2B), suggesting that proteasome inhibition induces DSBs by blocking the DNA synthesis.

Since Trx1 is an essential cofactor during cell proliferation, and given that DNA synthesis was decreased after proteasome inhibition, we decided to investigate the effects of MG132 on TRX1 gene expression. Analysis by RT-PCR revealed that TRX1 mRNA levels were not affected by sub-lethal doses of MG132 (figure 4A). However, Trx 1 protein levels decreased at $24 \mathrm{~h}$ (figure 4B), just when control cells should be 
proliferating (see figure $1 \mathrm{~A}$ and figure $3 \mathrm{C}$ ), and at $48 \mathrm{~h}$, when nuclear proteasome activation is required (see figure $3 \mathrm{~A}$, lower panel). In addition, we evaluated the subcellular localization of Trx1 $24 \mathrm{~h}$ after proteasome inhibition and found that MG132 induces Trx1 migration to the nucleus (figure 4C), suggesting that Trx1 may have a nuclear function after proteasome inhibition.

Proteasome inhibition directs Trx1 to the endoplasmic reticulum and prevent proteotoxic stress

In NH3T3 fibroblasts, proteasome inhibition with MG132 also induces the localization of Trx 1 in the extern membrane of the nuclear envelope. The Trx1 signal co-localizes with the endoplasmic reticulum at $24 \mathrm{~h}$ and $48 \mathrm{~h}$ after treatment, as judged by colocalization with the endoplasmic reticulum marker calnexin (figure 5A). This association of Trx1 to the ER may be directed to decrease the ER stress produced in the cells by the MG132 treatment. In fact, ER stress response markers such as BIP/Grp78, CHOP and PDI were overexpressed (Figure 5B) after 24 and $48 \mathrm{~h}$ of proteasome inhibition at low doses of MG132.

We study the activation of ER-associated proteotoxic stress pathway by measuring ATF4, BIM and BAX proteins. The overexpression of these proteins occurs after 24 anf 48h of proteasome inhibition (Figure $5 \mathrm{XX}$ ). 
Trx1 down-regulation by proteasome inhibition induces antioxidant response

Due to proteasome inhibition produces down-regulation of $\operatorname{Trx} 1$ and this protein is involved in the cellular redox regulation, we studied if the down-regulation of Trx1 by proteasome inhibition induces oxidative stress in the cells. The inhibition of proteasomes in NIH3T3 cells increased superoxide levels (Figure 6A), and also the GSSG/GSH ratio (Figure 6B) at 24 and $48 \mathrm{~h}$ after MG132 treatment.

Trx1 migration to the nucleus (Figure 4C) has been considered as a possible marker for oxidative stress [30], thus we wonder if antioxidant enzymes levels were affected by proteasome inhibition. Our western blot results indicate that proteasome inhibition at sub-lethal doses of MG132 stimulates the overexpression of the antioxidant enzymes catalase, CuZnSOD and MnSOD (Figure 6C). Thus, we suggest that Trx1 migration to the nucleus could be a mediator in the antioxidant response.

In order to complete the characterization of the antioxidant systems under conditions of proteasome inhibition, we analyzed the protein levels of the other members of the Trx and Grx family. Although an increase in Thioredoxin reductase (TrxR1) protein levels was found after proteasome inhibition (figure 6D), the mitochondrial and nuclear isoforms of Grx2 and the mitochondrial thioredoxin Trx2 were downregulated (Figure 6D). Thus, our results provide new information about the dependence of the function of Trx and the Grx family members on proteasome activity.

Trx1 levels and subcellular localization in a cell model with reduced proteasome activity: laforin-deficient fibroblasts from Lafora disease patients

To analyze the pathophysiological consequences of the relationship between proteasomes and Trx1, we choose fibroblasts from patients with Lafora disease with 
mutations in the EPM2A gene encoding the dual phosphatase laforin. First, we measured the three proteasome activities (chymotrypsin-, trypsin- and caspase-like) in these fibroblasts from two patients. The analysis was performed under high (starvation) and low (full medium) proteolysis (see Materials and Methods). Under low proteolysis (L), and although a tendency to down-regulation was observed, differences between controls and laforin-deficient cells were not significant (figure 7A). However, when high proteolysis conditions $(\mathrm{H})$ were induced, the chymotrypsin- and trypsin-like activities were lower in laforin-deficient fibroblasts (figure 7A). No differences were observed in the caspase-like activity in any of the conditions tested (figure 7A, right panel).

We also measured in pulse-chase experiments the degradation (total and proteasomedependent) of intracellular proteins. Results indicate that both total and proteasomedependent protein degradation was significantly decreased in laforin-deficient cells (figure 7B), corroborating the reduction in proteasome activity described above.

In order to confirm our hypothesis about the molecular relationship between Trx1 and proteasome, we decide to analyze Trx1 expression in fibroblasts from Lafora disease patients with mutations in the laforin gene.

As judged by RT-PCR analysis, laforin-deficient fibroblasts showed a lower TRX1 gene expression than control fibroblasts (figure 8A). In addition, the protein levels of Trx1 determined by Western blot were decreased in fibroblasts from Lafora patients compared to fibroblasts of healthy subjects (figure 8B). This reduction seems specific for the Trx1 protein since no differences were observed for the thioredoxin reductase 
protein TrxR1 (figure 8B). In addition, the reduction of Trx1 levels seems to be produced by low transcription of TRX1 gene.

We then analyzed the co-localization of Trx 1 with the 20S proteasome (figure 8C). Our results indicate that in control cells Trx1 was uniformly distributed in the cytosol, but with many foci in the nucleus. Interestingly, Trx 1 nuclear foci co-localized with the $20 \mathrm{~S}$ proteasome (figure $8 \mathrm{C}$, yellow dots in the nucleus), supporting our hypothesis for a Trx1 and 20S proteasome association, not only in the cytosol but also in the nucleus. However, in laforin deficient fibroblasts the distribution of Trx1 and the number of nuclear Trx1 foci were different, with a lower number of Trx1 foci in the nucleus and with Trx1 concentrated around the periphery of the nucleus, probably at the endoplasmic reticulum (ER) since Trx1 colocalized with calnexin (a marker for endoplasmic reticulum). The $20 \mathrm{~S}$ proteasome followed a similar pattern (figure $8 \mathrm{C}$, zoom), suggesting a common function for Trx1 and proteasomes at this subcellular domain (figure 8C) in fibroblasts from lafora disease patients.

As previously suggested, Trx1 migration to the nucleus has been considered as a possible marker for oxidative stress [30] and may mediate the antioxidant response in cells. Thus, we consider that control of antioxidant and UPR responses may be deregulated in Lafora fibroblasts.

\section{Discussion}

Trx1 is one of the most important antioxidant enzymes [31] and mediates other cellular events such as apoptosis [7] and regulation of transcription factors [6, 32, 33], among others. Its inhibition produces cell proliferation deregulation due to its importance for 
DNA synthesis [34]. On the other hand, the proteasome system is a central player for protein degradation and its inhibition has been shown to produce also cell proliferation inhibition, protein translation down-regulation, and sensitization to apoptosis in cells [4, 11]. Targeting protein degradation through the proteasome dependent pathway represents a novel approach for the treatment of cancer [35]. It has been recently shown that the use of the proteasome inhibitor MG132 induces apoptosis via oxidative stress in C6 glioma cells [36]. These effects may be produced in a time- and dose-dependent manner and, although the effects of proteasome inhibition have been studied, the mechanisms involved in cell growth inhibition after proteasome down-regulation are not fully elucidated.

Previous studies have proposed that $26 \mathrm{~S}$ proteasome was associated with thioredoxin proteins [37]. Furthermore, thioredoxin family members, like Trx1, Trx2 and Txnl1 were found associated with the $26 \mathrm{~S}$ proteasome in budding yeast $[19,38]$. In addition, proteasome subunits Rpt3 (26S regulatory subunit 6B) and Rpn12 (26S non-ATPase regulatory subunit 8) have been shown to be targets of thioredoxins in plant cells and in a mammalian neuroblastoma cell line [39-41]. However, the intriguing mechanisms underlying the relationship between thioredoxins and proteasome are not completely understood, even less in the nucleus of mammalian cells.

The thioredoxin related protein $(32 \mathrm{KDa})$ Txnll could act as redox sensor that mediates signals of the cell to the proteasome [20]. These findings reflect the essential mission that may exert the Trx system on proteasome function because its oxidation and/or glutathionylation affect the proteasome activity [42-44]. This relationship between Trx proteins and proteasome may attenuate oxidative stress conditions to avoid protein 
aggregation [44]. Thus, both systems may contribute to control cell proliferation and cell survival.

The cellular situations in which proteasome and Trx1 may work together remain unknown. Thus, in this work we describe the important events that occur in TRX1 expression and the changes in sub-cellular distribution and co-localization of the Trx 1 protein with the $20 \mathrm{~S}$ proteasome during cell proliferation or stress conditions, when proteasome was inhibited using sub-lethal doses of MG132 in NIH3T3 cells, a cell line in which cell cycle, redox profile and total and nuclear proteasome activity have been well characterized.

Trx1 protein levels in NIH3T3 were increased at $24 \mathrm{~h}$ (time of maximal DNA synthesis) and $48 \mathrm{~h}$ (time in which nuclear proteasome activity was increased, as we have previously shown [23]) (figure 1C). Our results suggest translational regulation of Trx1 during cell proliferation because mRNA levels of TRX1 gene were not affected during cell cycle. We wonder if Trx 1 co-localize with the $20 \mathrm{~S}$ proteasome during proliferation of NIH3T3 cells. Our results showed a similar pattern of cellular distribution for Trx 1 and 20S proteasome (figure 2), observing a perinuclear distribution for both proteins after seeding ( $6 \mathrm{~h})$ and a homogeneous distribution of Trx1 between nucleus and cytosol at 24 and $48 \mathrm{~h}$ of culture, just when cells were proliferating. However, when cells reached the confluence $(120 \mathrm{~h})$, both proteins left the nucleus and were completely redistributed in the cytosol (figure 2). All these results agree with the proteasome distribution reported by Palmer et al. [45] in other cell lines in which proteasome distributed to the nucleus during $\mathrm{S}$ phase, and redistributed towards cellular periphery during G2. Thus, we suggest that Trx1 and proteasomes may interact in mammalian cells, as it has been recently observed in yeast for Txnl1 protein [29]. 
To demonstrate this hypothesis we inhibited proteasome (cellular and nuclear proteasomes) using MG132 (figure 3A and figure 3B). Using sub-lethal doses of MG132, we blocked the DNA synthesis in NIH3T3 (figure 4C) in a process mediated by PARP-1 inhibition (figure 4D). These results agree with previous obtained by Awasthi N et al. [46] in HLE B-3 cells and in human lens epithelium explants, in which proteasome activity was demonstrated to be necessary for cell proliferation. But the most important observation was that Trx1 protein levels decreased at the cell proliferation times of $24 \mathrm{~h}$ and $48 \mathrm{~h}$ (figure 5B) when proteasome activity was inhibited, suggesting interdependence between proteasome and Trx1.

This interrelationship was not only between levels of Trx and proteasome, but also at sub-cellular localization of both proteins. In that way, we observed that after $24 \mathrm{~h}$ of proteasome inhibition, Trx1 concentrates into the nucleus (figure 5C), underscoring the idea that Trx1 is involved in important nuclear signaling events after proteasome inhibition.

It was demonstrated that Trx 1 migrates to the nucleus in cells under oxidative stress conditions [3, 47, 48]. In our experiments Trx 1 was also observed in ER at $24 \mathrm{~h}$ and 48 $\mathrm{h}$ after inhibition of proteasome (Figure 5A). The distribution of Trx1 and calnexin (an ER marker) was mainly surrounding the nuclei, suggesting a possible role of Trx 1 in the ER. In fact, analysis of ER stress markers, clearly showed that MG132 treatment induced overexpression of BIP, CHOP and PDI indicating ER stress conditions (Figure 5B) $[49,50]$. Furthermore, our results showed that superoxide levels (figure 6A) and GSSG/GSH ratio (figure 6B) were increased after MG132 treatment, suggesting that proteasome inhibition at sub-lethal doses induces oxidative stress. 
Furthermore, ATF4 protein was detected after proteasome inhibition indicating the activation of proteotoxic stress.

Provided that Trx1 is an essential component in the cellular antioxidant response and its translocation to the nucleus is necessary for transcription factors that contain regulatory cysteines in their DNA binding region, such as nuclear factor (erythroid-derived 2)-like 2 (Nrf-2) involved in antioxidant enzymes expression [51, 52], we decided to evaluate cellular antioxidant defenses. We observed that catalase, CuZnSOD and MnSOD were up-regulated (figure 6C), as it was previously described in other cell lines by Meiners et al. [53]. Probably this up-regulation was induced to maintain the redox equilibrium in the cell, suggesting that migration of Trx1 to the nucleus may favor the expression of these antioxidant enzymes.

Thioredoxin reductase, TrxR1, was also over-expressed after MG132 treatment (figure 6D), suggesting that the Cys residues of Trx 1 are oxidized in agreement with the observed GSSG/GSH ratio. A similar mechanism has been described in S. cerevisiae [54]. However, other thiol systems were differentially affected after proteasome inhibition with MG132. The mitochondrial form Trx2, and Grx2, the mitochondrial and nuclear variant of glutaredoxin, were also down-regulated after proteasome inhibition (figure 6D). Grx accepts electrons from GSH and from TrxR1 [55]. Thus, the mammalian thiol-enzymatic systems may be seriously affected after proteasome inhibition at sub-lethal doses of MG132.

To further study the molecular interdependence between Trx1 and proteasome we decide to analyze these systems in Lafora disease fibroblasts. In these cells, it has been described that proteasomal activity is reduced. Thus, ubiquitinated proteins and proteasome subunits accumulate in Lafora bodies [16, 56]. However, it is not 
completely clear if these conditions can produce oxidative stress in the patients, and it is not know what happens with Trx systems. Thus, fibroblasts from Lafora disease patients with mutations in the EPM2A gene encoding laforin are an excellent model to evaluate the expression of Trxland its sub-cellular localization in conditions in which proteasome activity is compromised.

In agreement with these studies, we observed in our laforin-deficient cells under high proteolysis conditions, low proteasomal chymotrypsin-like and trypsin-like activities (figure 7A) and also decreased degradation of intracellular proteins by proteasomes (figure 7B). In addition, as we show in figure 8, analysis of laforin-deficient fibroblasts from two Lafora disease patients indicate that TRX1 gene expression and Trx1 protein levels were clearly lower than in the control cell lines (figure 8B), the protein levels were also decreased in NIH3T3 when proteasome was inhibited. In that case, low protein levels are consequence of the down-regulation of TRX1 expression and may be affected in diseases in which proteasome activity was impaired. The analysis of Trx1 and $20 \mathrm{~S}$ proteasome localization in both, control and laforin deficient fibroblasts, suggested a different distribution for Trx 1 in laforin-deficient and in control fibroblasts: Trx1 was uniformly distributed across the cytoplasm in control fibroblasts (figure 8C) while it was concentrated at the nuclear periphery in laforin-deficient cells (figure 8C). We analyzed the co-localization of Trx 1 with the $20 \mathrm{~S}$ proteasome and we observed areas with high intensity of both proteins around the nuclear periphery, suggesting that Trx 1 and proteasome may cooperate in a common process (figure $8 \mathrm{C}$ ), to facilitate the degradation of oxidized proteins by the proteasome or to recover proteasome activity by reducing cysteine residues, as it has been suggested for yeast [57]. 
In addition, we found co-localization of Trx 1 with the ER marker, calnexin (figure 8D). Recent results indicated that Trx1 mediates responses after ER stress [58], and it was described that related members of Trx family are involved in the homeostasis of ER [31]. Under basal conditions, the analysis of expression of ER stress responsive proteins, like CHOP, BIP, including the Trx-family related protein PDI, did not show significant changes in laforin-deficient fibroblasts compared to control cells (data not shown). These results are in agreement with those previous obtained by Vernia et al. [16] in which these proteins did not change their levels unless the cells were incubated with ER stressors (e.g. thapsigargin). That results differ from those observed for NIH3T3, and suggest differences in cell physiology in Lafora fibroblasts. That results points out the possibility that Trx1 could exert a protective role mediating an antioxidant mission in the ER and, in fact, Vernia et al. [16], described the role of laforin/malin complex as protective proteins in ER-stress. It is known that prolonged conditions of ER-stress triggers apoptosis and brain samples of human LD patients lacking laforin showed increased levels of ER-stress markers. Previous results demonstrate that ER-stress induction by 2-deoxyglucose induces high levels of apoptosis in cells defective in laforin [59]. Trx1 is also known as a protector against apoptosis [60]. In consequence, we suspect that the low levels of nuclear Trx1 in the nucleus of laforin deficient cells may contribute to an aggravation of this process. That hypothesis agrees with the apoptosis levels observed in NIH3T3 cells after proteasome inhibition, in which Trx1 concentration in the nucleus may attenuate the apoptosis in NIH3T3 (figure S2A).

Finally, our results indicate that proteasome activity regulates Trx1 protein levels. In addition, migration of Trx1 to the nucleus and ER may have an important mission, in 
order to protect these cellular domains involved in essential responses to the stress as antioxidant enzyme expression, DNA damage response (DDR) and unfolded protein response (UPR). Recent findings indicate the importance of the proteasome activity during the DDR pathway [61]. Trx1 and proteasome coordination seems to be important in DNA repairing processes because proteasome plays an essential role in the DDR following DNA damage induction, by recycling DNA interacting proteins [62-65]. Recently, we have also described that nuclear proteasome activation is important during DNA synthesis in order to remove the excess of histones in the cells [23]. Now, we describe the importance of Trx1 migration to the nucleus after proteasome inhibition in the activation of antioxidant and UPR responses in mammalian cells.

In conclusion, we propose that Trx 1 contributes to the optimal activity of proteasome and this interaction is very relevant in the nucleus and the ER of mammalian cells. Low levels of Trx1 linked to low amount of Trx1-20S foci observed in the nucleus of laforindeficient cells may contribute to the disease phenotype. Thus, analyzing Trx systems in diseases in which the proteasome pathway is altered may offer valuable information about the origin of oxidative stress or Trx mediated functions, in these diseases. 


\section{Conclusions}

A proper interaction between Trx 1 and proteasome in mammalian cells during cell cycle is essential for the physiological functions of cells. In addition, proteasome activity controls Trx1 protein levels and its sub-cellular distribution, contributing to the expression of antioxidant enzymes and proteins involved in the UPR.

Since Trx1 levels and its sub-cellular localization are related to proteasome function, analyzing Trx1 in diseases in which the proteasome pathway is deregulated, like occurs in Lafora disease, will offer valuable information about the origin of some of the features of these diseases. 


\section{Acknowledgements}

The authors wish to thank to S. Bañuls for their technical support. F.V. Pallardó, P. Sanz and Erwin Knecht are members of the Rare Disease Micro-cluster of VLC Campus of Excellence. This work was supported by grants SAF2008-01338 from the Ministerio de Ciencia e Innovación and financial support from the CIBERER (Biomedical Network Research Center for Rare Diseases). The CIBERER is an initiative of the Instituto de Salud Carlos III and INGENIO 2010.

\section{Author's contribution}

Conceived and designed the experiments: JL GG, FVP. Performed the experiments: MSC, JL GG, FD, CA, CRM, JM and SP. JLGG and MSC contributed equally to the experiments of this work. Analyzed the data: JLGG, CA, CRM, EK, PS and FVP. Contributed reagents/materials/analysis tools: FVP, EK, PS. Wrote the manuscript: JLGG, FVP, PS, EK. 


\section{List of Abbreviations}

ASK-1: Apoptosis signal-regulating kinase 1

BIP/Grp78: 78 kDa glucose-regulated protein

BrdU: Brome deoxyuridine

CHOP: C/EBP-homologous protein (DNA damage-inducible transcript 3 protein)

CuZnSOD: Cupper Zinc Superoxide dismutase

ER: endoplasmic reticulum

DDR: DNA damage response

DSB: double strand breaks

GRX: Glutaredoxin

GSH: reduced glutathione

GSSG: oxidized glutathione

MnSOD: Manganese Superoxide dismutase

PARP: poly(ADP-ribose) polymerase

PDI: Protein disulfide-isomerase

TRX1: Thioredoxin 1

TRXR1: Thioredoxin reductase 1

UPR: unfolded protein response 


\section{References}

[1] Holmgren, A. Thioredoxin. Annu Rev Biochem 54:237-271; 1985.

[2] Thelander, L.; Reichard, P. Reduction of ribonucleotides. Annu Rev Biochem 48:133$158 ; 1979$.

[3] Arai, R. J.; Masutani, H.; Yodoi, J.; Debbas, V.; Laurindo, F. R.; Stern, A.; Monteiro, H. P. Nitric oxide induces thioredoxin-1 nuclear translocation: possible association with the p21Ras survival pathway. Biochem Biophys Res Commun 348:1254-1260; 2006.

[4] Collet, J. F.; Messens, J. Structure, function, and mechanism of thioredoxin proteins. Antioxid Redox Signal 13:1205-1216; 2010.

[5] Akamatsu, Y.; Ohno, T.; Hirota, K.; Kagoshima, H.; Yodoi, J.; Shigesada, K. Redox regulation of the DNA binding activity in transcription factor PEBP2. The roles of two conserved cysteine residues. J Biol Chem 272:14497-14500; 1997.

[6] Hirota, K.; Matsui, M.; Iwata, S.; Nishiyama, A.; Mori, K.; Yodoi, J. AP-1 transcriptional activity is regulated by a direct association between thioredoxin and Ref-1. Proc Natl Acad Sci U S A 94:3633-3638; 1997.

[7] Saitoh, M.; Nishitoh, H.; Fujii, M.; Takeda, K.; Tobiume, K.; Sawada, Y.; Kawabata, M.; Miyazono, K.; Ichijo, H. Mammalian thioredoxin is a direct inhibitor of apoptosis signalregulating kinase (ASK) 1. EMBO J 17:2596-2606; 1998.

[8] Lu, J.; Holmgren, A. Thioredoxin system in cell death progression. Antioxid Redox Signal 17:1738-1747.

[9] Hershko, A.; Ciechanover, A.; Varshavsky, A. Basic Medical Research Award. The ubiquitin system. Nat Med 6:1073-1081; 2000.

[10] Hershko, A.; Ciechanover, A. The ubiquitin system. Annu Rev Biochem 67:425-479; 1998.

[11] Hochstrasser, M. Protein degradation or regulation: Ub the judge. Cell 84:813-815; 1996.

[12] Oh, S.; Hong, H. S.; Hwang, E.; Sim, H. J.; Lee, W.; Shin, S. J.; Mook-Jung, I. Amyloid peptide attenuates the proteasome activity in neuronal cells. Mech Ageing Dev 126:12921299; 2005.

[13] McNaught, K. S.; Jenner, P. Proteasomal function is impaired in substantia nigra in Parkinson's disease. Neurosci Lett 297:191-194; 2001.

[14] Kabashi, E.; Agar, J. N.; Taylor, D. M.; Minotti, S.; Durham, H. D. Focal dysfunction of the proteasome: a pathogenic factor in a mouse model of amyotrophic lateral sclerosis. $J$ Neurochem 89:1325-1335; 2004.

[15] Zhou, H.; Cao, F.; Wang, Z.; Yu, Z. X.; Nguyen, H. P.; Evans, J.; Li, S. H.; Li, X. J. Huntingtin forms toxic $\mathrm{NH2}$-terminal fragment complexes that are promoted by the age-dependent decrease in proteasome activity. J Cell Biol 163:109-118; 2003.

[16] Vernia, S.; Rubio, T.; Heredia, M.; Rodriguez de Cordoba, S.; Sanz, P. Increased endoplasmic reticulum stress and decreased proteasomal function in lafora disease models lacking the phosphatase laforin. PLoS One 4:e5907; 2009.

[17] Low, P. The role of ubiquitin-proteasome system in ageing. Gen Comp Endocrinol 172:39-43; 2011.

[18] Zhang, P.; Wang, C.; Gao, K.; Wang, D.; Mao, J.; An, J.; Xu, C.; Wu, D.; Yu, H.; Liu, J. O.; $\mathrm{Yu}, \mathrm{L}$. The ubiquitin ligase itch regulates apoptosis by targeting thioredoxin-interacting protein for ubiquitin-dependent degradation. J Biol Chem 285:8869-8879; 2010.

[19] Andersen, K. M.; Madsen, L.; Prag, S.; Johnsen, A. H.; Semple, C. A.; Hendil, K. B.; Hartmann-Petersen, R. Thioredoxin Txnl1/TRP32 is a redox-active cofactor of the $26 \mathrm{~S}$ proteasome. J Biol Chem 284:15246-15254; 2009. 
[20] Wiseman, R. L.; Chin, K. T.; Haynes, C. M.; Stanhill, A.; Xu, C. F.; Roguev, A.; Krogan, N. J.; Neubert, T. A.; Ron, D. Thioredoxin-related Protein 32 is an arsenite-regulated Thiol Reductase of the proteasome 19 S particle. J Biol Chem 284:15233-15245; 2009.

[21] Vichai, V.; Kirtikara, K. Sulforhodamine B colorimetric assay for cytotoxicity screening. Nat Protoc 1:1112-1116; 2006.

[22] Livak, K. J.; Schmittgen, T. D. Analysis of relative gene expression data using real-time quantitative PCR and the 2(-Delta Delta C(T)) Method. Methods 25:402-408; 2001.

[23] Garcia-Gimenez, J. L.; Ledesma, A. M.; Esmoris, I.; Roma-Mateo, C.; Sanz, P.; Vina, J.; Pallardo, F. V. Histone carbonylation occurs in proliferating cells. Free Radic Biol Med 52:1453$1464 ; 2012$.

[24] Peterson, G. L. A simplification of the protein assay method of Lowry et al. which is more generally applicable. Anal Biochem 83:346-356; 1977.

[25] Fuertes, G.; Villarroya, A.; Knecht, E. Role of proteasomes in the degradation of shortlived proteins in human fibroblasts under various growth conditions. Int J Biochem Cell Biol 35:651-664; 2003.

[26] Fuertes, G.; Martin De Llano, J. J.; Villarroya, A.; Rivett, A. J.; Knecht, E. Changes in the proteolytic activities of proteasomes and lysosomes in human fibroblasts produced by serum withdrawal, amino-acid deprivation and confluent conditions. Biochem J 375:75-86; 2003.

[27] Markovic, J.; Borras, C.; Ortega, A.; Sastre, J.; Vina, J.; Pallardo, F. V. Glutathione is recruited into the nucleus in early phases of cell proliferation. J Biol Chem 282:20416-20424; 2007.

[28] Shan, W.; Zhong, W.; Zhao, R.; Oberley, T. D. Thioredoxin 1 as a subcellular biomarker of redox imbalance in human prostate cancer progression. Free Radic Biol Med 49:2078-2087; 2010.

[29] Andersen, K. M.; Jensen, C.; Kriegenburg, F.; Lauridsen, A. M.; Gordon, C.; HartmannPetersen, R. Txl1 and Txc1 are co-factors of the $26 \mathrm{~S}$ proteasome in fission yeast. Antioxid Redox Signal 14:1601-1608; 2011.

[30] Byrne, B. M.; Welsh, J. Altered thioredoxin subcellular localization and redox status in MCF-7 cells following 1,25-dihydroxyvitamin D3 treatment. J Steroid Biochem Mol Biol 97:5764; 2005.

[31] Wu, C.; Parrott, A. M.; Fu, C.; Liu, T.; Marino, S. M.; Gladyshev, V. N.; Jain, M. R.; Baykal, A. T.; Li, Q.; Oka, S.; Sadoshima, J.; Beuve, A.; Simmons, W. J.; Li, H. Thioredoxin 1-mediated post-translational modifications: reduction, transnitrosylation, denitrosylation, and related proteomics methodologies. Antioxid Redox Signal 15:2565-2604; 2011.

[32] Hirota, K.; Murata, M.; Sachi, Y.; Nakamura, H.; Takeuchi, J.; Mori, K.; Yodoi, J. Distinct roles of thioredoxin in the cytoplasm and in the nucleus. A two-step mechanism of redox regulation of transcription factor NF-kappaB. J Biol Chem 274:27891-27897; 1999.

[33] Makino, Y.; Yoshikawa, N.; Okamoto, K.; Hirota, K.; Yodoi, J.; Makino, I.; Tanaka, H. Direct association with thioredoxin allows redox regulation of glucocorticoid receptor function. J Biol Chem 274:3182-3188; 1999.

[34] Holmgren, A. Hydrogen donor system for Escherichia coli ribonucleoside-diphosphate reductase dependent upon glutathione. Proc Natl Acad Sci U S A 73:2275-2279; 1976.

[35] Wu, W. K.; Cho, C. H.; Lee, C. W.; Wu, K.; Fan, D.; Yu, J.; Sung, J. J. Proteasome inhibition: a new therapeutic strategy to cancer treatment. Cancer Lett 293:15-22; 2010.

[36] Fan, W. H.; Hou, Y.; Meng, F. K.; Wang, X. F.; Luo, Y. N.; Ge, P. F. Proteasome inhibitor MG-132 induces C6 glioma cell apoptosis via oxidative stress. Acta Pharmacol Sin 32:619-625; 2011.

[37] Liu, C. W.; Millen, L.; Roman, T. B.; Xiong, H.; Gilbert, H. F.; Noiva, R.; DeMartino, G. N.; Thomas, P. J. Conformational remodeling of proteasomal substrates by PA700, the $19 \mathrm{~S}$ regulatory complex of the $26 \mathrm{~S}$ proteasome. J Biol Chem 277:26815-26820; 2002. 
[38] Guerrero, C.; Milenkovic, T.; Przulj, N.; Kaiser, P.; Huang, L. Characterization of the proteasome interaction network using a QTAX-based tag-team strategy and protein interaction network analysis. Proc Natl Acad Sci U S A 105:13333-13338; 2008.

[39] Ishii, T.; Sakurai, T.; Usami, H.; Uchida, K. Oxidative modification of proteasome: identification of an oxidation-sensitive subunit in $26 \mathrm{~S}$ proteasome. Biochemistry 44:13893$13901 ; 2005$.

[40] Wong, J. H.; Balmer, Y.; Cai, N.; Tanaka, C. K.; Vensel, W. H.; Hurkman, W. J.; Buchanan, B. B. Unraveling thioredoxin-linked metabolic processes of cereal starchy endosperm using proteomics. FEBS Lett 547:151-156; 2003.

[41] Yamazaki, D.; Motohashi, K.; Kasama, T.; Hara, Y.; Hisabori, T. Target proteins of the cytosolic thioredoxins in Arabidopsis thaliana. Plant Cell Physiol 45:18-27; 2004.

[42] Demasi, M.; Silva, G. M.; Netto, L. E. 20 S proteasome from Saccharomyces cerevisiae is responsive to redox modifications and is S-glutathionylated. J Biol Chem 278:679-685; 2003.

[43] Grune, T.; Jung, T.; Merker, K.; Davies, K. J. Decreased proteolysis caused by protein aggregates, inclusion bodies, plaques, lipofuscin, ceroid, and 'aggresomes' during oxidative stress, aging, and disease. Int J Biochem Cell Biol 36:2519-2530; 2004.

[44] Silva, G. M.; Netto, L. E.; Simoes, V.; Santos, L. F.; Gozzo, F. C.; Demasi, M. A.; Oliveira, C. L.; Bicev, R. N.; Klitzke, C. F.; Sogayar, M. C.; Demasi, M. Redox control of $20 \mathrm{~S}$ proteasome gating. Antioxid Redox Signal 16:1183-1194; 2012.

[45] Palmer, A.; Mason, G. G.; Paramio, J. M.; Knecht, E.; Rivett, A. J. Changes in proteasome localization during the cell cycle. Eur J Cell Biol 64:163-175; 1994.

[46] Awasthi, N.; Wagner, B. J. Suppression of human lens epithelial cell proliferation by proteasome inhibition, a potential defense against posterior capsular opacification. Invest Ophthalmol Vis Sci 47:4482-4489; 2006.

[47] Wei, S. J.; Botero, A.; Hirota, K.; Bradbury, C. M.; Markovina, S.; Laszlo, A.; Spitz, D. R.; Goswami, P. C.; Yodoi, J.; Gius, D. Thioredoxin nuclear translocation and interaction with redox factor-1 activates the activator protein-1 transcription factor in response to ionizing radiation. Cancer Res 60:6688-6695; 2000.

[48] Spielberger, J. C.; Moody, A. D.; Watson, W. H. Oxidation and nuclear localization of thioredoxin-1 in sparse cell cultures. J Cell Biochem 104:1879-1889; 2008.

[49] Ma, Y.; Brewer, J. W.; Diehl, J. A.; Hendershot, L. M. Two distinct stress signaling pathways converge upon the CHOP promoter during the mammalian unfolded protein response. J Mol Biol 318:1351-1365; 2002.

[50] Imaoka, S. Chemical stress on protein disulfide isomerases and inhibition of their functions. Int Rev Cell Mol Biol 290:121-166; 2011.

[51] Hansen, J. M.; Watson, W. H.; Jones, D. P. Compartmentation of Nrf-2 redox control: regulation of cytoplasmic activation by glutathione and DNA binding by thioredoxin-1. Toxicol Sci 82:308-317; 2004.

[52] Kim, Y. C.; Yamaguchi, Y.; Kondo, N.; Masutani, H.; Yodoi, J. Thioredoxin-dependent redox regulation of the antioxidant responsive element (ARE) in electrophile response. Oncogene 22:1860-1865; 2003.

[53] Meiners, S.; Ludwig, A.; Lorenz, M.; Dreger, H.; Baumann, G.; Stangl, V.; Stangl, K. Nontoxic proteasome inhibition activates a protective antioxidant defense response in endothelial cells. Free Radic Biol Med 40:2232-2241; 2006.

[54] Tan, S. X.; Greetham, D.; Raeth, S.; Grant, C. M.; Dawes, I. W.; Perrone, G. G. The thioredoxin-thioredoxin reductase system can function in vivo as an alternative system to reduce oxidized glutathione in Saccharomyces cerevisiae. J Biol Chem 285:6118-6126; 2010.

[55] Johansson, C.; Lillig, C. H.; Holmgren, A. Human mitochondrial glutaredoxin reduces Sglutathionylated proteins with high affinity accepting electrons from either glutathione or thioredoxin reductase. J Biol Chem 279:7537-7543; 2004. 
[56] Rao, S. N.; Maity, R.; Sharma, J.; Dey, P.; Shankar, S. K.; Satishchandra, P.; Jana, N. R. Sequestration of chaperones and proteasome into Lafora bodies and proteasomal dysfunction induced by Lafora disease-associated mutations of malin. Hum Mol Genet 19:4726-4734; 2010. [57] Silva, G. M.; Netto, L. E.; Discola, K. F.; Piassa-Filho, G. M.; Pimenta, D. C.; Barcena, J. A.; Demasi, M. Role of glutaredoxin 2 and cytosolic thioredoxins in cysteinyl-based redox modification of the 20S proteasome. FEBS J 275:2942-2955; 2008.

[58] Luo, F. C.; Zhou, J.; Lv, T.; Qi, L.; Wang, S. D.; Nakamura, H.; Yodoi, J.; Bai, J. Induction of endoplasmic reticulum stress and the modulation of thioredoxin-1 in formaldehyde-induced neurotoxicity. Neurotoxicology 33:290-298; 2012.

[59] Wang, Y.; Liu, Y.; Wu, C.; McNally, B.; Zheng, P. Laforin confers cancer resistance to energy deprivation-induced apoptosis. Cancer Res 68:4039-4044; 2008.

[60] Nadeau, P. J.; Charette, S. J.; Landry, J. REDOX reaction at ASK1-Cys250 is essential for activation of JNK and induction of apoptosis. Mol Biol Cell 20:3628-3637; 2009.

[61] Kouranti, I.; Peyroche, A. Protein degradation in DNA damage response. Semin Cell Dev Biol; 2012.

[62] Daulny, A.; Tansey, W. P. Damage control: DNA repair, transcription, and the ubiquitinproteasome system. DNA Repair (Amst) 8:444-448; 2009.

[63] Ben-Aroya, S.; Agmon, N.; Yuen, K.; Kwok, T.; McManus, K.; Kupiec, M.; Hieter, P. Proteasome nuclear activity affects chromosome stability by controlling the turnover of Mms22, a protein important for DNA repair. PLoS Genet 6:e1000852; 2010.

[64] Cabrera, R.; Sha, Z.; Vadakkan, T. J.; Otero, J.; Kriegenburg, F.; Hartmann-Petersen, R.; Dickinson, M. E.; Chang, E. C. Proteasome nuclear import mediated by Arc3 can influence efficient DNA damage repair and mitosis in Schizosaccharomyces pombe. Mol Biol Cell 21:3125-3136; 2010.

[65] Levy-Barda, A.; Lerenthal, Y.; Davis, A. J.; Chung, Y. M.; Essers, J.; Shao, Z.; van Vliet, N.; Chen, D. J.; Hu, M. C.; Kanaar, R.; Ziv, Y.; Shiloh, Y. Involvement of the nuclear proteasome activator PA28gamma in the cellular response to DNA double-strand breaks. Cell Cycle 10:4300-4310; 2011. 


\section{Figure legends}

Figure 1. Measurement of Trx 1 mRNA and protein levels during cell proliferation of NIH3T3 fibroblasts A) DNA synthesis in NIH3T3 fibroblasts measured by the BrdU incorporation assay. B) Trx1 mRNA levels measured at different times of cell culture by RT-PCR. Results of BrdU incorporation are the mean and S.D. from two separate experiments with triplicated samples and Trx 1 mRNA levels represent the mean and S.D. from two separate experiments with duplicated samples. Stars indicate statistically significant differences from control values at $* p<0.05$. C) Trx1 protein levels determined at different times of cell culture by Western blot.

Figure 2. Sub-cellular localization by immunofluorescence of $\operatorname{Trx} 1$ and the $20 \mathrm{~S}$ proteasome in NIH3T3 fibroblasts after $6 \mathrm{~h}, 24 \mathrm{~h}, 48 \mathrm{~h}$ and $120 \mathrm{~h}$ of cell culture. Images show the sub-cellular distribution of Trx1 (red) and of the 20S proteasome (green) and the merge images for each time.

Figure 3. Effects of the proteasome inhibitor MG132 at $0.2 \mu \mathrm{M}$ in NIH3T3 fibroblasts. A) Total (upper panel) and nuclear (lower panel) chymotrypsin-like activity was measured after 24 and $48 \mathrm{~h}$ of cell culture in presence or absence of MG132. B) Western blot of poly-ubiquitinated proteins after 24 and $48 \mathrm{~h}$ of cell culture in the presence or absence of MG132. C) DNA synthesis evaluated by the BrdU incorporation assay after 24 and $48 \mathrm{~h}$ of cell culture in the presence or absence of MG132. D) Analysis of PARP levels by Western blot after MG132 treatment at 24 and 48h of cell culture. Results of chymotrypsin-like activity and BrdU incorporation represent the mean and S.D. from two separate experiments with triplicated samples. Stars indicate statistically significant differences from control values at $* \mathrm{p}<0.05$. 
Figure 4. Changes in Trx1 levels and its cellular localization after incubation of NIH3T3 fibroblasts with MG132. A and B) Trx1 mRNA levels evaluated by RT-PCR (A) and Western blot analysis of Trx1 (B) in NIH3T3 control and MG132 treated fibroblasts after 24 and $48 \mathrm{~h}$ of cell culture. C) Localization of Trx 1 evaluated by immunofluorescence and confocal microscopy after proteasome inhibition with MG132 and $24 \mathrm{~h}$ of cell culture. Arrows indicate nuclear concentration of Trx1. Trx 1 mRNA levels represent the mean and S.D. from two separate experiments with triplicated samples. Stars indicate statistically significant differences from control values at $* \mathrm{p}<0.05$.

Figure 5. Localization of Trx1 in the ER and expression of unfolded protein response chaperones. A) Co-localization of Trx1 with the ER marker calnexin evaluated by confocal microscopy. B) Effect of proteasome inhibition with MG132 on the levels of the chaperones BIP (Brp78), CHOP and PDI at 24 and $48 \mathrm{~h}$ of cell culture. Arrows indicate localization of Trx1 in the ER.

Figure 6. Changes in the redox profile and in the expression of antioxidant enzymes of NIH3T3 cells levels after MG132 treatment. A) Superoxide levels. B) GSSG/GSH ratio. C and D) Western blot analysis of the levels of antioxidant enzymes catalase, CuZnSOD and MnSOD (C) and of Trx2, TrxR1 and Grx2 (D) in NIH3T3 treated with $0.2 \mu \mathrm{M}$ MG132 for 24 and $48 \mathrm{~h}$. Results of superoxide levels and GSSG/GSH ratio represent the mean and S.D. from two separate experiments with triplicated samples. Stars indicate statistically significant differences from control values at $* \mathrm{p}<0.05$.

Figure 7. Proteasomes are impaired in laforin-deficient fibroblasts. A) Chymotripsin-, trypsin- and caspase-like activities were measured in extracts from control and laforindeficient fibroblasts under high $(\mathrm{H})$ and low (L) proteolysis conditions as described in 
Materials and Methods. Results are shown as the percentage relative to the control value under high proteolysis conditions and represent the mean and S.D. from three to five separate experiments with triplicated samples. B) Human fibroblasts from controls and from patients of Lafora disease were labelled with $\left[{ }^{3} \mathrm{H}\right]$ valine and the total and proteasome-dependent degradation of long-lived proteins under high proteolysis conditions was measured and calculated as described in Materials and Methods. Results represent the percentage of the labelled protein that is degraded per $\mathrm{h}$ and are the mean and S.D. from five to seven separate experiments with duplicated samples. Stars indicate statistically significant differences from control values at $* p<0.05, * * p<0.005$ and $* * * p<0.0005$

Figure 8. Analysis of Trx1 levels and cellular distribution in control and in fibroblasts from Lafora's patients fibroblasts. A) mRNA levels for TRX1 gene in fibroblasts from controls ( $\mathrm{C} 1$ and $\mathrm{C} 2)$ and from patients of Lafora disease (L1 and L2) were analyzed by RT-PCR. B) Western blot analysis Trx1 and TrxR1 levels C and D) Cellular distribution of Trx1 and 20S proteasomes (C) and Trx1 and the ER marker calnexin (D) evaluated by confocal microscopy. Results of mRNA levels represent the mean and S.D. from two separate experiments with duplicated samples. Stars indicate statistically significant differences from control values at $* \mathrm{p}<0.05$. Arrows indicate co-localization of Trx1 and 20S proteasomes in the cytoplasm and the nucleus. 


\section{Supplementary figures}

Figure S1. Effect of an MG132 treatment on the survival and morphology of NIH3T3 cells. A) $\mathrm{IC}_{50}$ determination by the sulforhodamine B assay. B) Cell morphology.

Figure S2. Evaluation of the apoptosis in NIH3T3 fibroblasts after incubation with MG132 for 24 and 48 h. A) Analysis of the apoptosis with Annexin V/propidium iodide and AMNIS technology. B) Western blot analysis of the levels of gamma-H2AX. 